Beresford T.P., Schwartz, J., Wilson., D., et al (1992) The short term psychological health of alcoholic and nonalcoholic liver transplant recipients. Alcoholism: Clinical and Experimental Research, 16, 996-1000.

Colus, I., Burroughs, A., Rolles, K., et al (1995) Psychiatric and social outcome of liver transplantation. British Journal of Psychiatry, 166, 521-524.

Howard, L., FAHY, T., Wong, P., et al (1994) Psychiatric outcome in alcoholic liver transplant patients. Quarterly Journal of Medicine, 87, 731-736.

LOWE, D., O'Grady, J., McEwan, J., et al (1990) Quality of life following liver transplantation: a preliminary report. Journal of the Royal College of Physicians, 24, 43-46.

L.M. HOWARD

T. FAHY

The Maudsley Hospital

London SE5 8AZ

\section{Costs of community psychiatric nurse teams}

SIR: McCrone et al's paper (August 1994) analyses data from the Greenwich service reported in the preceding paper in the same issue by Muijen et al and concludes that "... the CST [community support team] is a cost-effective alternative to generic CPN [community psychiatric nurse team] arrangements". Over the 18 months studied the generic group is claimed to cost an average of $£ 110$ more per patient per week.

Close examination of the data does not appear to support this conclusion. This small study demonstrated remarkably few differences in either clinical and social outcome or in reduction in hospital care despite markedly increased CPN contact in the intervention group. Where, then, do the cost savings arise?

The major cost advantage to the CST group is accounted for by lower accommodation costs $£ 148$ per patient per week as opposed to $£ 269$ for the generic group. This is presumably due to the higher number of generic patients who were living in specialist care settings ( $22 \%$ c.f. $2 \%$ at intake and $22 \%$ c.f. $3 \%$ at follow up according to Muijen et al). The figures are harder to disentangle in the McCrone et al paper but they state - "More clients from the generic group lived in specialist care settings (homes, hostels or hospital) both at referral $(15 \%)$ and 18 months later (23\%)." Direct treatment costs, on the other hand, are marginally greater in the CST group (i.e. subtracting accommodation costs from total costs) - $£ 137$ per patient per week c.f. $£ 126$ in generic care.

There is no reason to assume from these two papers that the differences in accommodation costs are anything other than an artefact of the randomisation. The failure to acknowledge this and make adequate allowances for it in the paper's discussion obscures the study findings. McCrone et al have conducted their economic analysis following Beecham \& Knapp's (1990) four principles of cost evaluation (which emphasise the need for comprehensives). These four principles are probably essential for costing across widely differing procedures and disorders (e.g. comparing the cost benefits of hip replacements against diabetic out-patient clinics). Their application in RCTs of a defined patient population, however, obscures more than it illuminates.

Judgement needs to be exercised in the conduct of economic evaluations in mental health studies if they are not to lead to serious misunderstandings as I believe they have in this paper.

BeECHAM, J. \& KNAPP, M. (1990) Costing mental health services. Psychological Medicine, 20, 893-908.

McCrone. P., BeECham, J. \& KNAPP, M. (1994) Community psychiatric nurse teams: cost-effectiveness of intensive support versus generic care. British Journal of Psychiatry. 165, 218-221. Muden, M., CoOney, M., Strathdee, G., et al (1994) Community psychiatric nurse teams: intensive support versus generic care. British Journal of Psychiatry, 165, 211-217.

\section{St Georges Hospital Medical School London SW17 ORE}

AUTHOR's REPLY: Burns has confused the shortterm (0-6 months) and longer-term (0-18 months) findings from our cost-effectiveness study of the Greenwich service.

The quote from our paper in his first paragraph is taken out of context. The words which precede the clause he quotes are "In the short term, therefore ...". And the sentence which follows the quote is: "Beyond the short term, the CST did not have a cost or cost-effectiveness advantage". We categorically did not say that the CST was more cost-effective than generic CPN services over the 18-month period.

What happened in the short term (0-6 months) to give the significant cost advantage to the CST? Accommodation and hospital costs were significantly lower. When account is taken of the fact that the CST group looked as if it made use of less specialised accommodation in the pre-referral period, we still find the CST to have a cost advantage in the first six months of the intervention. ('Net costs' between pre-referral and 0-6 months showed CST costs were still lower than generic costs; $P<0.05)$. Over the whole research period (0-18 months), accommodation costs were lower for the CST group $(P<0.001)$, but other costs counter-balanced this advantage to give the 